\title{
Erratum to: Recent Advances in Asymmetric Nitroso Diels-Alder Reactions
}

\author{
Xuan-Bo Xu ${ }^{a}$, Chen-Fu Liu ${ }^{b}$, and Guo-Wu Rao ${ }^{a}$ * \\ ${ }^{a}$ College of Pharmaceutical Science and Institute of Drug Development \& Chemical Biology, \\ Zhejiang University of Technology, Hangzhou, China \\ *e-mail: rgw@zjut.edu.cn \\ ${ }^{b}$ School of Pharmaceutical Sciences, Gannan Medical University, Ganzhou, China \\ Received May 25, 2019
}

DOI: $10.1134 / \mathrm{S} 1070428019060265$

The second author should be Chen-Fu Liu.

The original article can be found online at https://doi.org/10.1134/S1070428019040237. 